\title{
Chaoization of Switched Reluctance Motor Drives
}

\author{
J.F. Huang ${ }^{1}$, J.H. Chen ${ }^{1}$, and K.T. Chau ${ }^{2}$ \\ ${ }^{1}$ Department of Electrical Engineering, Tsinghua University, Beijing 100084, P. R. of China \\ ${ }^{2}$ Department of Electrical and Electronic Engineering, The University of Hong Kong, Hong Kong, P.R. of China
}

\begin{abstract}
This paper presents a new technique for the chaoiztion of switched reluctance motor (SRM) drivers. Based on the chaotic modeling of SRM drives, effects of feedback controller gains on the stability of the rotor speed are investigated. In accordance, a control strategy combining piecewise proportional feedback and time-delayed feedback is proposed to produce bound-controllable oscillation around any specific rotor speed. To estimate the continuous influence of a certain control parameter, a Poincaré map sampling at every extreme points is constructed, then the bifurcation diagrams are drawn. Theoretical analysis and numerical simulation for a practical $12 / 8$ motor driver are also given.
\end{abstract}

\section{INTRODUCTION}

Recently, a number of research activities on chaos in motor drives have been carried out. Most of them are based on the identification of chaos, the avoidance of chaos and the stabilization of chaos in various types of electric motor drives [1-6]. Many methods deriving form the idea of parameter disturbing, namely OGY method, are employed to stabilize an aperiodic or chaotic rotor speed into a periodic or regular steady state [7-9]. However, in some industrial occasions chaotic motions in motor drives are highly desired to improve the effectiveness and efficiency, for example, mixing and grinding. Such tries, so-called anti-control of chaos or chaoization have been barely reported. In this paper, rather than negatively avoiding the occurrence of chaos in motors, a positive idea to utilize the chaotic motion for some niche applications will be presented.

Recent experiences have proved that conventional controllers can also induce chaos in motor drivers. This paper firstly presents the analysis of the effect of parameters of the controller on the stability of dynamic system. It can be shown that some chaotic behaviors potentially offer distinct advantages of the reduction of subharmonics, electromagnetic interference (EMI) and acoustic noise. However, this kind of chaotic motion suffers a limited range of oscillation of the rotor speed. To amplify its chaotic domain, a new control technique, named time-delayed feedback control, has been combined with the modified proportional feedback. This novel controller has the advantages that only parameters need to adjust and no inherent parameter of the motor is necessary to change. It can be easily implemented with digital controller and has high flexibility. Despite of the benefits above, time-delayed feedback control bring some obstacles into the analysis and computation. To facilitate the controller design, the switching effect should be ignored and hence, other methods to estimate its stability must be proposed.

In section II, the modeling of chaos in switched reluctance motor (SRM) drives is described without ignoring the switching effect nor accepting rough assumptions. In section III, based on the modeling of chaos, the chaotic operation with lower ripples of the torque and rotor speed is realized. In section IV, with ignoring the switching effect, the modeling of chaoization in SRM drives is derived for a controllable chaotic motion. In section $\mathrm{V}$, to estimate the continuous influence of a certain control parameter, the Poincaré map is constructed; then the bifurcation diagrams are proposed and analyzed.

\section{Modelling of Chaos in Switched Reluctance MOTOR DRIVES}

Due to the double salient structure of switched reluctance motors, nonlinear phenomena, like fringing and saturation, are severe during the time the rotor and stator are overlapped. So the flux linkage is related with both phase current and rotor position. The most common modeling of flux linkage, current and rotor position is a smooth surface interpolated by second-order Lagrange interpolation functions using data measured experimentally or computed by finite element method.

\section{A. State Equations}

Fig. 1 shows the schematic diagram of a three-phase SRM which is adopted for exemplification.

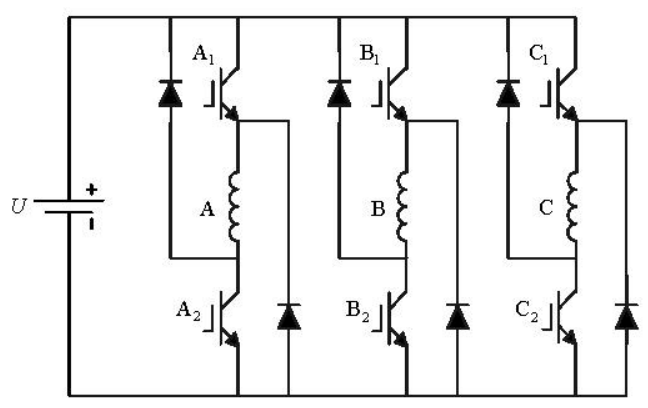

Fig. 1 Schematic diagram of SRM drives.

Since SRM phase windings are conducted in turn, only two adjacent phase windings have currents at the same instant given the dwell angle $\theta_{d}$ of every phase winding equal to the commutation angle $\theta_{s}$. In order to 
simplify the calculation of the Poincare map, the rotor position $\theta$ rather than time $t$ is selected as the independent variable of the system. Hence, the system equation becomes [11]:

$$
\left\{\begin{aligned}
\frac{d \omega}{d \theta} & =\left(-B \omega+T_{e}\left(\theta, \Psi_{1}^{\prime}, \Psi_{2}^{\prime}, \theta_{s}\right)-T_{l}\right) /(J \omega) \\
\frac{d \Psi_{1}^{\prime}}{d \theta} & =\left(-R i_{1}\left(\theta, \Psi_{1}\right)+u_{1}(\theta)\right) / \omega \\
\frac{d \Psi_{2}^{\prime}}{d \theta} & =\left(-R i_{2}\left(\theta-\theta_{s}, \Psi_{2}^{\prime}\right)+u_{2}\left(\theta-\theta_{s}\right)\right) / \omega \\
T_{e} & =-\frac{\partial}{\partial \theta} \int_{0}^{\Psi_{1}} i_{1}\left(\theta, \Psi_{1}\right) d \Psi_{1}^{\prime}-\frac{\partial}{\partial \theta} \int_{0}^{\Psi_{2}} i_{2}\left(\theta-\theta_{s}, \Psi_{2}\right) d \Psi_{2}
\end{aligned}\right.
$$

where $\omega$ is the rotor speed, $i_{k}\left(\theta, \Psi_{k}\right)$ and $\Psi_{k}\left(\theta, i_{k}\right)(k=1$, 2 ) are the $k$ th phase current and flux linkage, $R$ is the phase resistance, $B$ is the viscous damping, $J$ is the load inertia, $T_{l}$ is the load torque.

\section{B. Control Equations}

For each conductive phase winding, the corresponding current control is achieved by applying a current hysteresis controller, as Fig. 2.

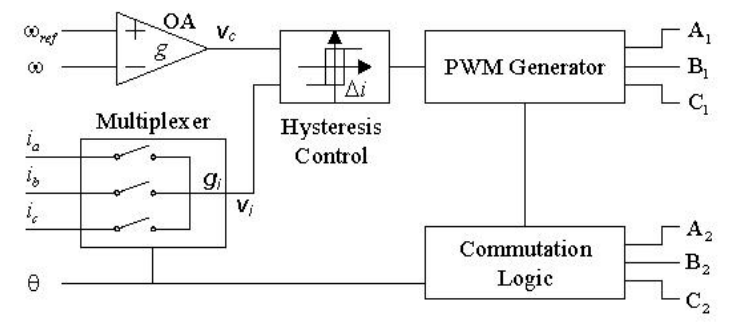

Fig. 2 SRM hysteresis controller.

The current reference signal $v_{c}(\theta)$, the current control signal $v_{i}(\theta)$ and the hysteresis band signal are given as follows:

$$
\left\{\begin{aligned}
v_{c}(\theta) & =k_{p}\left(\omega_{c}-\omega(\theta)\right)+\int k_{i}\left(\omega_{c}-\omega(\theta)\right) d \theta \\
v_{i}(\theta) & =g_{i} i_{k}(\theta) \\
\Delta v & =g_{i} \Delta i
\end{aligned}\right.
$$

$\omega_{c}$ is the reference speed. The current hysteresis controller outputs the signal to turn on or off those upper-leg power switches (A1, B1 and $\mathrm{C} 1$ ), depending on the phase commutation. When $\left(v_{i}-v_{i}\right)>\Delta v$, the upper-leg switch being the same phase of the turn-on lower-leg switch is off until $\left(v_{i}-v_{i}\right)<-\Delta v$, then it is on.

\section{Chaolzation of Switched Reluctance MotoR DRIVES}

Changing system parameters such as $k_{p}, k_{i}$ and $g_{i}$, the appearances and qualities of solutions can be quite different, varying from fundamental solution to subharmonic, even chaos. In this paper, parameters from a practical 12/8 SRM and its controller have been used to simulate the real operation numerically.
TABLE I.

SIMULATION PARAMETERS

\begin{tabular}{|l|l|l|l|}
\hline$N_{s}$ & 12 & $N_{r}$ & 8 \\
\hline$\theta_{s}$ & $15^{\circ}$ & $\theta_{s}$ & $15^{\circ}$ \\
\hline$\theta_{o}$ & $5.5^{\circ}$ & $R$ & $0.1 \Omega$ \\
\hline$J$ & $0.025 \mathrm{kgm}^{2}$ & $B$ & $0.005 \mathrm{Nm} / \mathrm{rads}^{-1}$ \\
\hline$U_{s}$ & $150 \mathrm{~V}$ & $\omega_{c}$ & $50 \mathrm{rads}^{-1}$ \\
\hline$\Delta i$ & $2 \mathrm{~A}$ & $g_{i}$ & $0.02 \Omega$ \\
\hline$k_{p}$ & $1 \mathrm{~V} / \mathrm{rads}^{-1}$ & $k_{i}$ & $0.01 \mathrm{~V} / \mathrm{rads}^{-1}$ \\
\hline$T_{l}$ & $12 \mathrm{Nm}$ & & \\
\hline
\end{tabular}

When proportional gain $k_{i}$ is $1,10,22 \mathrm{~V} / \mathrm{rads}^{-1}$ respectively, the corresponding trajectories of torque versus rotor speed are shown as Fig. 3-5. It indicates that the system exhibits fundamental, subharmonic and chaotic operations

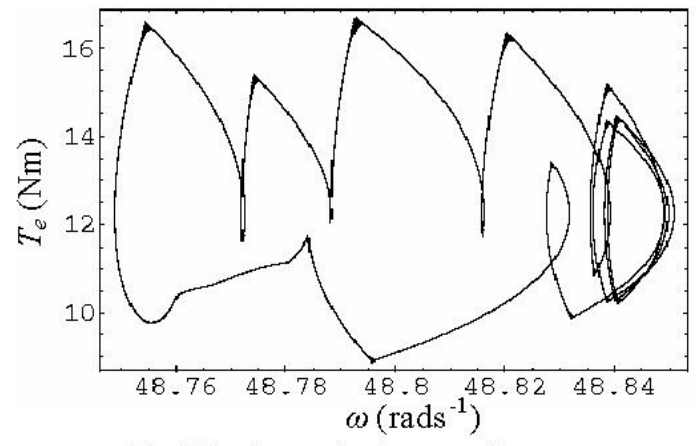

Fig. 3 Fundamental trajectory of $T_{e}$ versus $\omega$.

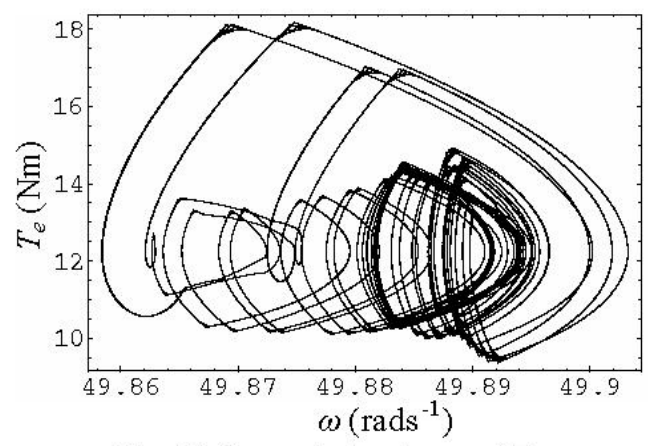

Fig. 4 Subharmonic-4 trajectory of $T_{e}$ versus $\omega$.

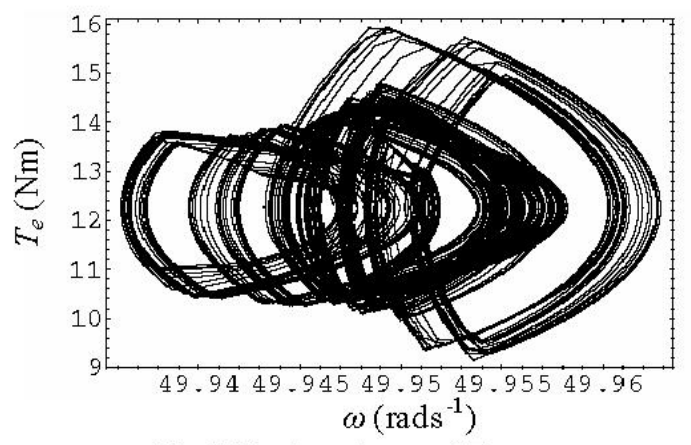

Fig. 5 Chaotic trajectory of $T_{e}$ versus $\omega$. 
The Waveforms and power spectra $P_{T}$ of the fundamental and chaotic electromagnetic torques $T_{e}$ are respectively shown in Fig. 6 and 7. Due to the broad frequency band of the chaotic operation, the later torque has more uniform distribution in the view of power spectrum. Comparing Fig. 6(b) and 7(b), they can be found that chaotic motion can reduce the annoying peaks during commutation, and hence reduce the abrasion and noise.

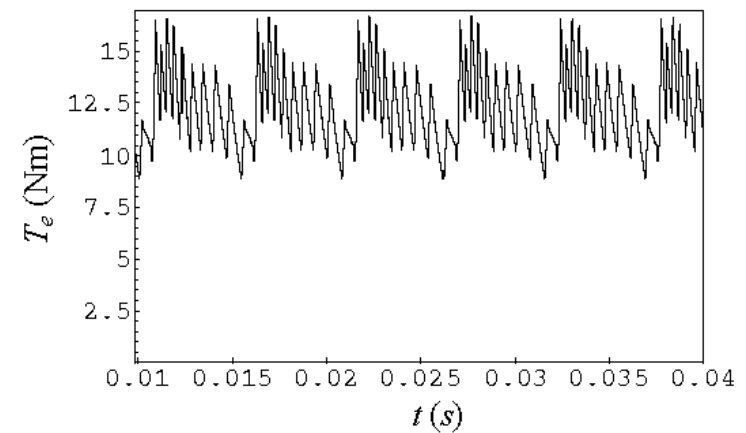

(a)

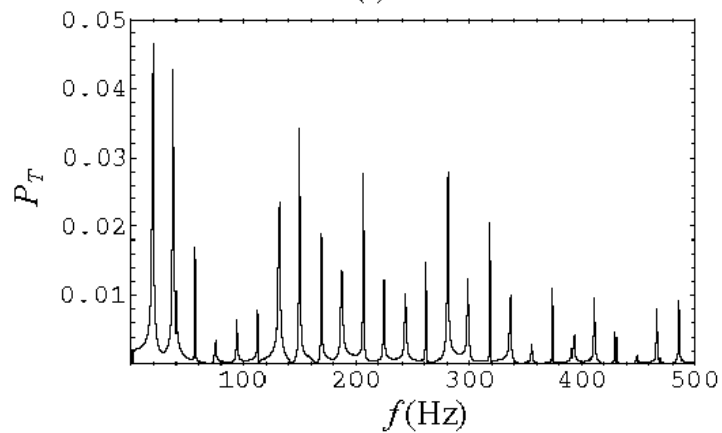

(b)

Fig. $6 \mathrm{~W}$ aveform and power spectrum of the fundamental solution: (a) Waveform of torque; (b) Power spectrum.

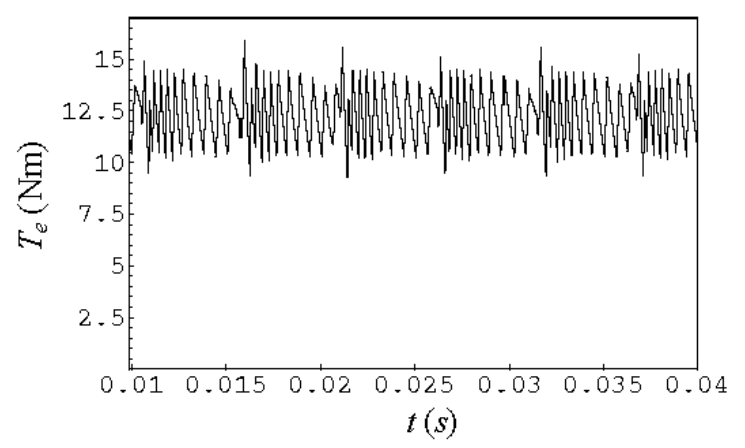

(a)

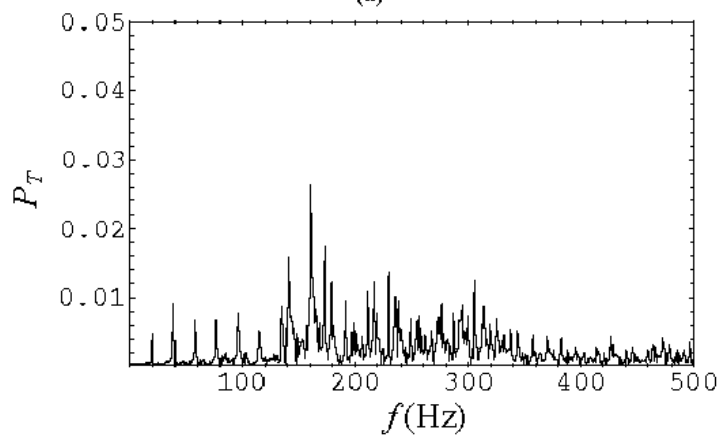

(b)

Fig. 7 Waveform and power spectrum of the chaotic solution:

(a) Waveform of torque; (b) Power spectrum.

\section{Chaolzation Combining TIME-Delayed AND PIECEWISE PROPORTIONAL FEEDBACK}

As shown above, proportional gains restrain the variation range of the rotor speed and prohibit the motor to oscillate in a large area though it can in fact improve the frequency of oscillation. To obtain the required oscillation in a desired range, other control techniques must be used. Time-delayed feedback can transform an ordinary differential equation into an infinitedimensional differential equation, and therefore has the advantage of introducing infinite kinds of dynamical characteristics into the original systems [10].

We add a sinusoidal function of the state variable into the reference signal:

$$
\begin{aligned}
v_{c}(\theta)=k_{p}\left(\omega_{c}-\omega(\theta)\right)+\int k_{i}\left(\omega_{c}-\omega(\theta)\right) d \theta \\
+\mu \operatorname{Sin}\left(\sigma\left(\omega_{c}-\omega(\theta-\tau)\right)\right.
\end{aligned}
$$

In the meantime, the proportional gain $k_{p}$ has also been modified as following. When the rotor speed lies right in the desired range, no proportional feedback is added to the reference signal, which means that the system is overwhelmed by time-delayed feedback control. Once the rotor speed leaves the desired domain, the linear feedback will pull it back.

$$
k_{p}(\omega)= \begin{cases}-\Delta \omega_{c}+\omega_{c}-\omega & \omega-\omega_{c}<-\Delta \omega_{c} \\ 0 & \left|\omega-\omega_{c}\right|<\Delta \omega_{c} \\ \Delta \omega_{c}+\omega_{c}-\omega & \omega-\omega_{c}>\Delta \omega_{c}\end{cases}
$$

Setting a desired range for the rotor speed, such as $[48,52] \mathrm{rads}^{-1}$, different performances of the rotor speed can be regulated by changing the magnitude and frequency of the sinusoidal function and the delay time as well. When the frequency $\sigma$ is set with 1 and 3 respectively, the system operates in the periodic and chaotic motions as show in Fig. 8 and 9. It can be found that the oscillation boundaries of both periodic and chaotic rotor speed, namely $[49,53] \mathrm{rads}^{-1}$, are near to the desired rang. It indicates that the system has a controllable chaotic motion. As shown in Fig. 8, it is interesting that the periodic solution is actually a heteroclinic trajectory which has two unstable saddle points locating respectively at $\omega=47.5 \mathrm{rads}^{-1}$ and $\omega$ $=52.5 \mathrm{rads}^{-1}$.

It should be noted that the commutation angle has been chosen as the unit for rotor position because much wider oscillation of rotor speed in spatial and temporal concept are what are now focused on.

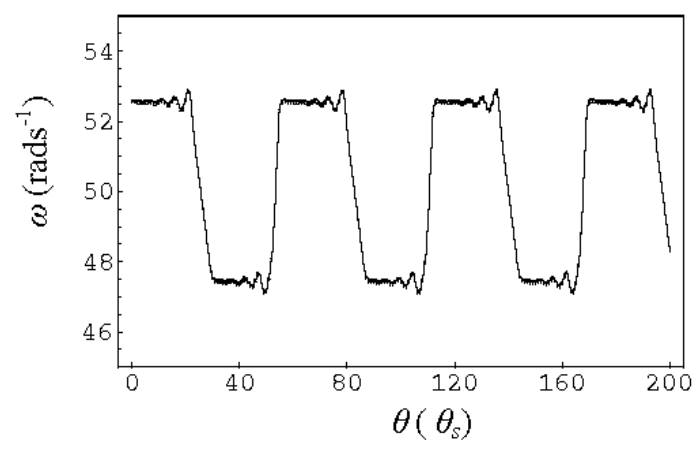

Fig. 8 Waveform of periodic solution. 


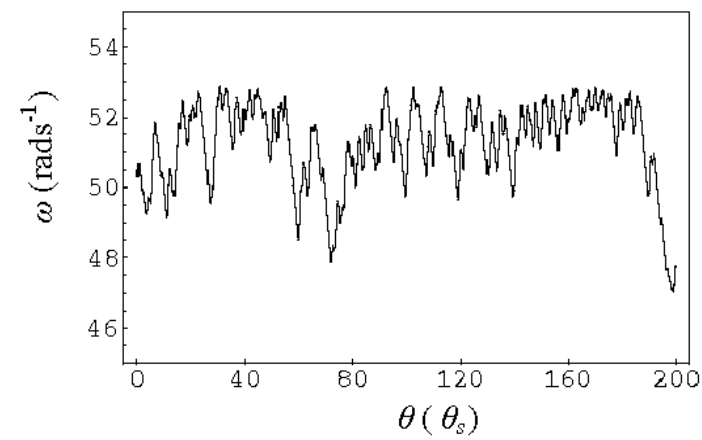

Fig. 9 Waveform of chaotic solution.

\section{SIMPLIFIED DESIGN MODELLING}

When the rotor speed oscillating in a much wider range in spatial and temporal concept is the main object to be considered, the minor variations caused by PWM switching operation can often be ignored without affecting the result much. Another reason to give up the original analysis modeling is its high order and heavy computation time. Assume the phase current follows the reference signal quick enough; the state equations can be simplified as a second-order differential equation:

$$
\left\{\begin{array}{l}
\frac{d \theta}{d t}=\omega \\
\frac{d \omega}{d t}=\left(-B \omega+T_{e}(\theta, i)-T_{l}\right) / J
\end{array}\right.
$$

Assume the phase current stays constant in a sampling period, the discretized control equation can be write as:

$$
\begin{aligned}
i_{c}(n)=g_{i}\left[k_{p}\left(\omega_{c}-\omega(n)\right)+\sum_{m=1}^{n} k_{i}\left(\omega_{c}-\omega(m)\right)\right. \\
+\mu \operatorname{Sin}\left(\sigma\left(\omega_{c}-\omega(n-N)\right)\right]
\end{aligned}
$$

In order to analyze the influence of a system parameter, the bifurcation diagrams should be gained with other parameters fixed and changing the chosen parameter continuously. Construction of Poincare map is a prerequisite to draw bifurcation diagrams. Here, a new method based on extremum points to construct Poincaré map is proposed. Select the Poincaré section as:

$$
\Sigma:=\{(\theta, \omega): \dot{\omega}(t)=0\}
$$

Hence, the Poincaré map $P: R^{2} \rightarrow R^{2}$ is defined as:

$$
\mathbf{X}_{n+1}=P\left(\mathbf{X}_{n}\right)
$$

The correspondence between the continuous waveform of the rotor speed and its discrete mapping points derived from Poincaré map is illustrated with exemplification of the chaotic motion as shown in Fig. 10. If the mapping has two fixed points, which means a maximum point and a minimum point, it indicates that the motor operates in periodic solution; if the motor speed is like the waveform in Fig. 10, then when the system is stable a set of dense points will be got instead of two points.

Keep other parameters as listed in the table 1, and vary the $\mu$ and $k_{p}$, the corresponding bifurcation diagrams can be achieved as shown in Fig. 11 and Fig. 12. Increasing $\mu$, the rotor speed transfers from periodic solution into chaos as shown in Fig. 11. Contrary to the Fig. 11, increasing $k_{p}$, the rotor speed transfers from chaos into periodic solution as shown in Fig. 12. It indicates that the dynamic characteristic depends on the balance of the sinusoidal function of time-delayed feedback and the proportional feedback gain. It can also be found that the boundaries of the chaotic rotor speed for the different parameter $\mu$ and $k_{p}$ always keep in the same range. Hence, the chaotic range is controllable. Furthermore, since no periodic window exists in chaotic parameter areas, this chaotic behavior is robust.

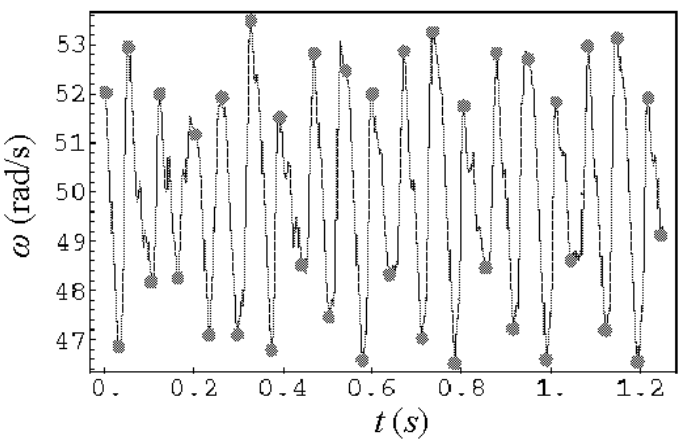

Fig. 10 Illustration of Poincaré map.

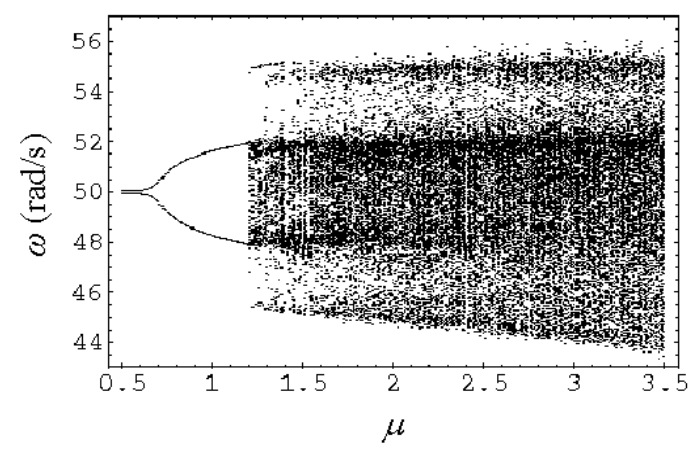

Fig. 11 Bifurcation of rotor speed versus $\mu$.

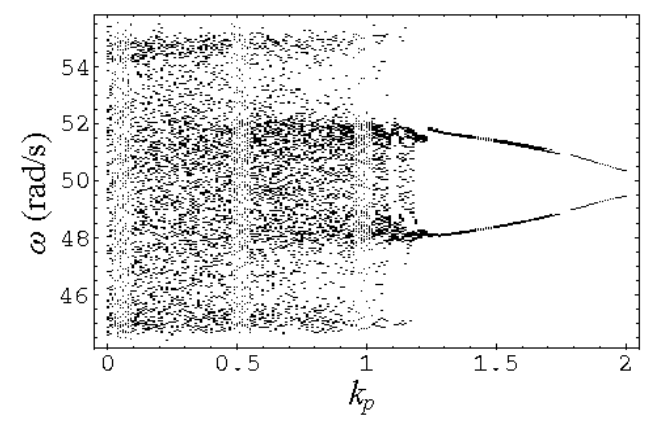

Fig. 12 Bifurcation of rotor speed versus $k_{p}$.

\section{CONCLUSION}

In this paper, the effects of parameter variations in a conventional controller on the stability of switched reluctance motor drives have been firstly investigated. It can be seen that some chaotic behaviors potentially offer distinct advantages of reduction of subharmonics, EMI and acoustic noise. Secondly a feedback control technique combining with time-delayed feedback and piecewise proportional feedback is proposed to achieve desired bound-controllable and robust chaotic motion. In despite of its benefits, delayed feedback control increase the computation tremendously. In order to reduce the computation time, a simplified chaotic design modeling 
has been derived with the assumption of the current loop following the rotor speed loop quick enough. Meantime, a method to construct Poincaré map based on extrema sampling has been presented. The bifurcation diagrams show that when a certain parameter of the controller varies continuously, the dynamic system exhibits a sudden bifurcation into chaos.

\section{ACKNOWLEDGMENT}

This work was supported and funded by National Natural Science Foundation of China (50177015).

\section{REFERENCES}

[1] N. Hemati, "Strange attractors in brushless do motors," IEEE Transactions on Circuits and Systems - I, Vol. 41, 1994, pp. 40-45.

[2] T. Asakura, et al., "Chaos detection in velocity control of induction motor and its control by using neural network," Proc. of IEEE ICSP, 2000, pp. 1633-1638.

[3] Chen, J.H., et al., "Chaos in voltage-mode controlled do drive system," International Journal of Electronics, 1999, Vol. 86, No. 7, p. 857-874.

[4] Zhong Li, et al., "Bifurcations and chaos in a permanent-magnet synchronous motor," IEEE Transactions on Circuits and Systems $-I$, Vol. 49, 2002, pp.383-387.

[5] Y. Gao, K.T. Chau, "Design of permanent magnets to avoid chaos in PM synchronous machines," IEEE Transactions on Magnetics, Vol. 39, No. 5, 2003, pp.2995-2997.

[6] Reiss, J., et al., "A detailed analysis of the nonlinear dynamics of the electric step motor Industrial Technology," 2002. IEEE ICIT '02. 2002 IEEE International Conference on, Volume: 2, 11-14 Dec. 2002 Pages: 1078 - 1083 vol.2.

[7] Ito, Shunji, et al., "Abrasive machining under wet condition and constant pressure using chaotic rotation," Seimitsu Kogaku Kaishi/Journal of the Japan Society for Precision Engineering, Vol. 64, No. 5, 1998, pp748-752.

[8] J. Guckenheimer, et al., Nonlinear oscillations, Dynamical Systems, and Birfucations of Vector Fields, (New York: Springer-Verlag), 1983.

[9] Parker T.S, Chua L O, Practical Numerical Algorithms for Chaotic Systems, (New York: Springer-Verlag), 1989.

[10] G. Chen, et al., From Chaos to Order: Methodologies, Perspectives and Applications, Singapore: World Scientific, 1998.

[11] K.T. Chau, J.H. Chen, “ Modeling, analysis, and experimentation of chaos in a switched reluctance drive system” , IEEE Transactions on Circuits and SystemsI: Fundamental Theory and Applications, May 2003, Vol. 50, No. 5, pp712-716. 